\title{
CURVATURE PROPERTIES OF 3-QUASI-SASAKIAN MANIFOLDS
}

\author{
BENIAMINO CAPPELLETTI MONTANO, ANTONIO DE NICOLA, AND IVAN YUDIN
}

\begin{abstract}
We find some curvature properties of 3-quasi-Sasakian manifolds which are similar to some well-known identities holding in the Sasakian case. As an application, we prove that any 3-quasi-Sasakian manifold of constant horizontal sectional curvature is necessarily either 3 - $\alpha$-Sasakian or 3 -cosymplectic.
\end{abstract}

\section{INTRODUCTION}

An important topic in contact Riemannian geometry is the study of curvature properties of almost contact metric manifolds (see [1] for details). In some cases it is in fact possible to characterize a manifold in terms of its curvature tensor field. The typical example is given by Sasakian manifolds, which are characterized by the well-known condition

$$
R_{X Y} \xi=\eta(Y) X-\eta(X) Y .
$$

A key role in this area is played by the interaction between the curvature and the structure tensors $(\phi, \xi, \eta)$ of an almost contact metric manifold. For instance, in any Sasakian manifold one has

$$
\begin{aligned}
R(X, Y, Z, W)= & R(X, Y, \phi Z, \phi W)-g(X, Z) g(Y, W)+g(X, W) g(Y, Z) \\
& +g(X, \phi Z) g(Y, \phi W)-g(X, \phi W) g(Y, \phi Z)
\end{aligned}
$$

and in any cosymplectic manifold

$$
R(X, Y, Z, W)=R(X, Y, \phi Z, \phi W)
$$

for any vector fields $X, Y, Z, W$. The relations (1.2) and (1.3) turn out to be useful for studying the $\phi$-sectional curvature and the Ricci tensor and deriving other properties on the geometry of the manifold. A generalization of (1.2) and (1.3) was proposed by Janssens and Vanecke in [9]. They defined a $C(\alpha)$-manifold as a normal almost contact metric manifold whose curvature tensor satisfies the condition

$$
\begin{aligned}
R(X, Y, Z, W)= & R(X, Y, \phi Z, \phi W)+\alpha(-g(X, Z) g(Y, W)+g(X, W) g(Y, Z) \\
& +g(X, \phi Z) g(Y, \phi W)-g(X, \phi W) g(Y, \phi Z)),
\end{aligned}
$$

for some $\alpha \in \mathbb{R}$. $C(\alpha)$-manifolds include Sasakian, cosymplectic and Kenmotsu manifolds. Another generalization, due to Blair, is given by the notion of quasiSasakian structure ([2]). By definition, a quasi-Sasakian manifold is a normal almost contact metric manifold whose fundamental 2 -form $\Phi:=g(\cdot, \phi \cdot)$ is closed. This class includes Sasakian and cosymplectic manifolds and can be viewed as an odd-dimensional counterpart of Kähler structures. Although quasi-Sasakian manifolds were studied by several different authors and are considered a well-established

\footnotetext{
2000 Mathematics Subject Classification. Primary 53C12, Secondary 53C25, 57R30.

Key words and phrases. quasi-Sasakian, 3-quasi-Sasakian, 3- $\alpha$-Sasakian, 3-Sasakian, 3cosymplectic.

Research partially supported by CMUC and FCT (Portugal), through European program COMPETE/FEDER, grants PTDC/MAT/099880/2008 and PEst-C/MAT/UI0324/2011, MTM2009-13383 (A.D.N.), and SFRH/BPD/31788/2006 (I.Y.).
} 
topic in contact Riemannian geometry, only little about their curvature properties is known. With this regard we mention the attempts of Olszak ([10]) and Rustanov ([12]). On the other hand, if a quasi-Sasakian manifold is endowed with two additional quasi-Sasakian structures defining a 3-quasi-Sasakian manifold then, as shown in [3] and [4], the quaternionic-like relations force the three structures to satisfy more restrictive geometric conditions.

Motivated by these considerations, in this paper we study the curvature properties of 3-quasi-Sasakian manifolds. We are able to find conditions similar to (1.1), (1.2), and (1.3) for the 3-quasi-Sasakian case. Moreover, we present one application of these properties by proving a formula relating the three $\phi$-sectional curvatures of a 3-quasi-Sasakian manifold. We then obtain that a 3-quasi-Sasakian manifold has constant horizontal sectional curvature if and only if it is either 3-c-Sasakian or 3 -cosymplectic. In the first case it is a space of constant curvature $c^{2} / 4$ and in the latter case it is flat. The last result extends to the quasi-Sasakian setting a famous theorem of Konishi ([7]).

\section{PRELiminaries}

A quasi-Sasakian manifold $(M, \phi, \xi, \eta, g)$ of dimension $2 n+1$ is said to be of rank $2 p$ (for some $p \leq n$ ) if $(d \eta)^{p} \neq 0$ and $\eta \wedge(d \eta)^{p}=0$ on $M$, and to be of rank $2 p+1$ if $\eta \wedge(d \eta)^{p} \neq 0$ and $(d \eta)^{p+1}=0$ on $M$ (cf. [2, 13]). It was proven in [2] that there are no quasi-Sasakian manifolds of (constant) even rank. Particular subclasses of quasi-Sasakian manifolds are c-Sasakian manifolds (usually called $\alpha$ Sasakian), which have rank $2 n+1$, and cosymplectic manifolds (rank 1) according to satisfy, in addition, $d \eta=c \Phi(c \neq 0)$ and $d \eta=0$, respectively. For $c=2$ we obtain the well-known Sasakian manifolds.

If on the same manifold $M$ there are given three distinct almost contact structures $\left(\phi_{1}, \xi_{1}, \eta_{1}\right),\left(\phi_{2}, \xi_{2}, \eta_{2}\right),\left(\phi_{3}, \xi_{3}, \eta_{3}\right)$ satisfying the following relations, for any even permutation $(\alpha, \beta, \gamma)$ of $\{1,2,3\}$,

$$
\begin{gathered}
\phi_{\gamma}=\phi_{\alpha} \phi_{\beta}-\eta_{\beta} \otimes \xi_{\alpha}=-\phi_{\beta} \phi_{\alpha}+\eta_{\alpha} \otimes \xi_{\beta}, \\
\xi_{\gamma}=\phi_{\alpha} \xi_{\beta}=-\phi_{\beta} \xi_{\alpha}, \quad \eta_{\gamma}=\eta_{\alpha} \circ \phi_{\beta}=-\eta_{\beta} \circ \phi_{\alpha},
\end{gathered}
$$

we say that $\left(\phi_{\alpha}, \xi_{\alpha}, \eta_{\alpha}\right), \alpha \in\{1,2,3\}$, is an almost contact 3 -structure. Then the dimension of $M$ is necessarily of the form $4 n+3$. This notion was introduced independently by Kuo ([8]) and Udriste ([14]). An almost 3-contact manifold $M$ is said to be hyper-normal if each almost contact structure $\left(\phi_{\alpha}, \xi_{\alpha}, \eta_{\alpha}\right)$ is normal.

In [8] Kuo proved that given an almost contact 3 -structure $\left(\phi_{\alpha}, \xi_{\alpha}, \eta_{\alpha}\right)$, there exists a Riemannian metric $g$ compatible with each of the three almost contact structure and hence we can speak of almost contact metric 3-structure. It is well known that in any almost 3 -contact metric manifold the Reeb vector fields $\xi_{1}, \xi_{2}, \xi_{3}$ are orthonormal with respect to the compatible metric $g$. Moreover, by putting $\mathcal{H}=\bigcap_{\alpha=1}^{3} \operatorname{ker}\left(\eta_{\alpha}\right)$ we obtain a codimension 3 distribution on $M$ and the tangent bundle splits as the orthogonal sum $T M=\mathcal{H} \oplus \mathcal{V}$, where $\mathcal{V}=\left\langle\xi_{1}, \xi_{2}, \xi_{3}\right\rangle$. The distributions $\mathcal{H}$ and $\mathcal{V}$ are called, respectively, horizontal and Reeb distribution.

A 3-quasi-Sasakian structure is an almost contact metric 3-structure such that each structure $\left(\phi_{\alpha}, \xi_{\alpha}, \eta_{\alpha}, g\right)$ is quasi-Sasakian. Remarkable subclasses are 3-Sasakian and 3-cosymplectic manifolds. Another subclass of 3-quasi-Sasakian structures is given by almost contact metric 3 -structures $\left(\phi_{\alpha}, \xi_{\alpha}, \eta_{\alpha}, g\right)$ such that each structure $\left(\phi_{\alpha}, \xi_{\alpha}, \eta_{\alpha}, g\right)$ is $c_{\alpha}$-Sasakian. It is proven in [6] that the non-zero constants $c_{1}, c_{2}$, $c_{3}$ must coincide. Therefore we speak of 3-c-Sasakian manifolds. Many results on 3-quasi-Sasakian manifolds were obtained in [3] and [4]. We collect some of them in the following theorem. 
Theorem 2.1 ([3, 4]). Let $\left(M, \phi_{\alpha}, \xi_{\alpha}, \eta_{\alpha}, g\right)$ be a 3-quasi-Sasakian manifold of dimension $4 n+3$. Then, for any even permutation $(\alpha, \beta, \gamma)$ of $\{1,2,3\}$, the Reeb vector fields satisfy

$$
\left[\xi_{\alpha}, \xi_{\beta}\right]=c \xi_{\gamma}
$$

for some $c \in \mathbb{R}$. Moreover, the 1 -forms $\eta_{1}, \eta_{2}, \eta_{3}$ have the same rank, called the rank of the 3-quasi-Sasakian manifold $M$. The rank of $M$ is 1 if and only if $M$ is 3 -cosymplectic and it is an integer of the form $4 l+3$, for some $l \leq n$, in the other cases. Furthermore, any 3-quasi-Sasakian manifold of rank $4 n+3$ is necessarily 3-c-Sasakian.

We point out that the constant $c$ in $(2.2)$ is zero if and only if the manifold is 3 -cosymplectic. Moreover, for any 3 -quasi-Sasakian manifold of rank $4 l+3$ one can consider the distribution

$$
\mathcal{E}^{4 m}:=\left\{X \in \mathcal{H} \mid i_{X} \eta_{\alpha}=0, i_{X} d \eta_{\alpha}=0 \text { for any } \alpha=1,2,3\right\} \quad(l+m=n)
$$

and its orthogonal complement $\mathcal{E}^{4 l+3}:=\left(\mathcal{E}^{4 m}\right)^{\perp}$. We will also consider the distribution $\mathcal{E}^{4 l}$ which is the orthogonal complement of $\mathcal{V}$ in $\mathcal{E}^{4 l+3}$. A remarkable property of 3 -quasi-Sasakian manifolds, which in general does not hold for a single quasi-Sasakian structure, is that both $\mathcal{E}^{4 l+3}$ and $\mathcal{E}^{4 m}$ are integrable and define Riemannian foliations with totally geodesic leaves. In particular it follows that $\nabla \mathcal{E}^{4 l+3} \subset \mathcal{E}^{4 l+3}$ and $\nabla \mathcal{E}^{4 m} \subset \mathcal{E}^{4 m}$.

All manifolds considered in the paper are assumed to be connected. The Spivak's conventions for the differential, the wedge product and the interior product are adopted.

\section{Main Results}

We recall that in any 3 -quasi-Sasakian manifold of rank $4 l+3$ for each $\alpha \in$ $\{1,2,3\}$ one defines two tensors $\psi_{\alpha}$ and $\theta_{\alpha}$ by

$$
\psi_{\alpha}:=\left\{\begin{array}{ll}
\phi_{\alpha}, & \text { on } \mathcal{E}^{4 l+3} \\
0, & \text { on } \mathcal{E}^{4 m}
\end{array} \quad \theta_{\alpha}:= \begin{cases}0, & \text { on } \mathcal{E}^{4 l+3} \\
\phi_{\alpha}, & \text { on } \mathcal{E}^{4 m}\end{cases}\right.
$$

Moreover we define $\Psi_{\alpha}(X, Y):=g\left(X, \psi_{\alpha} Y\right)$ and $\Theta_{\alpha}(X, Y):=g\left(X, \theta_{\alpha} Y\right)$ for all $X, Y \in \Gamma(T M)$. The tensors $\psi_{\alpha}$ and $\Psi_{\alpha}$ satisfy

$$
d \eta_{\alpha}=c \Psi_{\alpha}, \quad \nabla \xi_{\alpha}=-\frac{c}{2} \psi_{\alpha}
$$

(cf. [4, (4.8)] and [4, Theorem 4.3]). Since $\phi_{\alpha}=\psi_{\alpha}+\theta_{\alpha}$ one has that $\Phi_{\alpha}=\Psi_{\alpha}+\Theta_{\alpha}$. Consequently, due to (3.1), $\Psi_{\alpha}$ and $\Theta_{\alpha}$ are closed 2-forms. We start with a few lemmas. The first is immediate.

Lemma 3.1. In any 3-quasi-Sasakian manifold of rank $4 l+3$ one has,

$$
\begin{gathered}
g\left(\psi_{\alpha}^{2} X, Y\right)=g\left(X, \psi_{\alpha}^{2} Y\right), \\
\psi_{\alpha}^{3}=-\psi_{\alpha}, \\
\nabla \eta_{\alpha}=\frac{c}{2} \Psi_{\alpha} .
\end{gathered}
$$

Lemma 3.2. In any 3-quasi-Sasakian manifold of rank $4 l+3$ one has

$$
\left(\nabla_{X} \psi_{\alpha}\right) Y=\frac{c}{2}\left(\eta_{\alpha}(Y) \psi_{\alpha}^{2} X-g\left(\psi_{\alpha}^{2} X, Y\right) \xi_{\alpha}\right)
$$

Proof. Let $X \in \Gamma(T M)$. According to the orthogonal decomposition $T M=\mathcal{E}^{4 l+3} \oplus$ $\mathcal{E}^{4 m}$ we may distinguish the following two cases. (i) Assume $Y \in \Gamma\left(\mathcal{E}^{4 l+3}\right)$. Then, since $\nabla \mathcal{E}^{4 l+3} \subset \mathcal{E}^{4 l+3}$, we have $\left(\nabla_{X} \psi_{\alpha}\right) Y=\nabla_{X}\left(\psi_{\alpha} Y\right)-\psi_{\alpha} \nabla_{X} Y=\nabla_{X}\left(\phi_{\alpha} Y\right)-$ $\phi_{\alpha} \nabla_{X} Y=\left(\nabla_{X} \phi_{\alpha}\right) Y$. The assertion then follows from [4, (4.9)]. (ii) If $Y \in \Gamma\left(\mathcal{E}^{4 m}\right)$, 
then, as $\nabla \mathcal{E}^{4 m} \subset \mathcal{E}^{4 m}$, one has $\left(\nabla_{X} \psi_{\alpha}\right) Y=\nabla_{X}\left(\psi_{\alpha} Y\right)-\psi_{\alpha} \nabla_{X} Y=0$. On the other hand, by using (3.2) and $Y \in \Gamma\left(\mathcal{E}^{4 m}\right) \subset \operatorname{ker}\left(\eta_{\alpha}\right) \cap \operatorname{ker}\left(\psi_{\alpha}\right)$, one has

$$
\frac{c}{2}\left(\eta_{\alpha}(Y) \psi_{\alpha}^{2} X-g\left(\psi_{\alpha}^{2} X, Y\right) \xi_{\alpha}\right)=-\frac{c}{2} g\left(X, \psi_{\alpha}^{2} Y\right) \xi_{\alpha}=0 .
$$

By using (3.5) and (3.3) we get straightforwardly the following formula for $\nabla \psi_{\alpha}^{2}$.

Lemma 3.3. In any 3-quasi-Sasakian manifold of rank $4 l+3$ one has

$$
\left(\nabla_{X} \psi_{\alpha}^{2}\right) Y=\frac{c}{2}\left(\Psi_{\alpha}(X, Y) \xi_{\alpha}-\eta_{\alpha}(Y) \psi_{\alpha} X\right) .
$$

Theorem 3.4. In any 3-quasi-Sasakian manifold the following formula holds

$$
R_{X Y} \xi_{\alpha}=\frac{c^{2}}{4}\left(\eta_{\alpha}(X) \psi_{\alpha}^{2} Y-\eta_{\alpha}(Y) \psi_{\alpha}^{2} X\right) .
$$

Proof. If the manifold is 3-cosymplectic, i.e. $c=0$, the claim follows easily from the property that each $\xi_{\alpha}$ is parallel. Thus we can assume that $M$ has rank $4 l+3$. By using (3.1), (3.5), and (3.2), we have

$$
\begin{aligned}
R_{X Y} \xi_{\alpha} & =\frac{c}{2}\left(\nabla_{Y}\left(\psi_{\alpha} X\right)-\nabla_{X}\left(\psi_{\alpha} Y\right)+\psi_{\alpha}[X, Y]\right) \\
& =\frac{c}{2}\left(\left(\nabla_{Y} \psi_{\alpha}\right) X-\left(\nabla_{X} \psi_{\alpha}\right) Y\right) \\
& =\frac{c^{2}}{4}\left(\eta_{\alpha}(X) \psi_{\alpha}^{2} Y-g\left(\psi_{\alpha}^{2} Y, X\right) \xi_{\alpha}-\eta_{\alpha}(Y) \psi_{\alpha}^{2} X+g\left(\psi_{\alpha}^{2} X, Y\right) \xi_{\alpha}\right) \\
& =\frac{c^{2}}{4}\left(\eta_{\alpha}(X) \psi_{\alpha}^{2} Y-\eta_{\alpha}(Y) \psi_{\alpha}^{2} X\right) .
\end{aligned}
$$

Theorem 3.5. Let $M$ be a 3-quasi-Sasakian manifold of rank $4 l+3$. Then,

$$
\begin{aligned}
R_{X Y} \phi_{\alpha} Z-\phi_{\alpha} R_{X Y} Z= & \frac{c^{2}}{4}\left(\left(\Psi_{\alpha}\left(Y, \psi_{\alpha} Z\right)-\eta_{\alpha}(Y) \eta_{\alpha}(Z)\right) \psi_{\alpha} X-\left(\Psi_{\alpha}\left(X, \psi_{\alpha} Z\right)\right.\right. \\
& \left.-\eta_{\alpha}(X) \eta_{\alpha}(Z)\right) \psi_{\alpha} Y-\Psi_{\alpha}(Y, Z) \psi_{\alpha}^{2} X+\Psi_{\alpha}(X, Z) \psi_{\alpha}^{2} Y \\
& \left.+\left(\eta_{\alpha}(X) \Psi_{\alpha}(Y, Z)-\eta_{\alpha}(Y) \Psi_{\alpha}(X, Z)\right) \xi_{\alpha}\right) .
\end{aligned}
$$

Proof. The claim follows from a long computation using (3.5), (3.6) and (3.3).

Corollary 3.6. In any 3-quasi-Sasakian manifold of rank $4 l+3$ one has

$$
g\left(R_{X Y} \phi_{\alpha} Z, W\right)+g\left(R_{X Y} Z, \phi_{\alpha} W\right)=-P_{\alpha}(X, Y, Z, W),
$$

where $P_{\alpha}$ is the tensor defined by

$$
\begin{aligned}
P_{\alpha}(X, Y, Z, W)= & \frac{c^{2}}{4}\left(\Psi_{\alpha}(Y, Z) \Psi_{\alpha}\left(X, \psi_{\alpha} W\right)-\Psi_{\alpha}(X, Z) \Psi_{\alpha}\left(Y, \psi_{\alpha} W\right)\right. \\
& +\Psi_{\alpha}\left(Y, \psi_{\alpha} Z\right) \Psi_{\alpha}(X, W)-\Psi_{\alpha}\left(X, \psi_{\alpha} Z\right) \Psi_{\alpha}(Y, W) \\
& -\eta_{\alpha}(X) \eta_{\alpha}(W) \Psi_{\alpha}(Y, Z)-\eta_{\alpha}(Y) \eta_{\alpha}(Z) \Psi_{\alpha}(X, W) \\
& \left.+\eta_{\alpha}(Y) \eta_{\alpha}(W) \Psi_{\alpha}(X, Z)+\eta_{\alpha}(X) \eta_{\alpha}(Z) \Psi_{\alpha}(Y, W)\right) .
\end{aligned}
$$

Corollary 3.7. In any 3-quasi-Sasakian manifold of rank $4 l+3$ one has

$$
\begin{aligned}
g\left(R_{\phi_{\alpha} X \phi_{\alpha} Y} \phi_{\alpha} Z, \phi_{\alpha} W\right)= & \frac{c^{2}}{4}\left(g\left(R_{X Y} Z, W\right)+\Psi_{\alpha}(Z, X) \Psi_{\alpha}\left(W, \psi_{\alpha} \phi_{\alpha} Y\right)\right. \\
& +\Psi_{\alpha}\left(Z, \psi_{\alpha} X\right) \Psi_{\alpha}\left(W, \phi_{\alpha} Y\right) \\
& +\Psi_{\alpha}\left(\phi_{\alpha} X, Z\right) \Psi_{\alpha}\left(\phi_{\alpha} Y, \psi_{\alpha} \phi_{\alpha} W\right) \\
& \left.+\Psi_{\alpha}\left(\phi_{\alpha} X, \psi_{\alpha} Z\right) \Psi_{\alpha}\left(\phi_{\alpha} Y, \phi_{\alpha} W\right)\right)
\end{aligned}
$$

for any $X, Y, Z, W \in \Gamma(\mathcal{H})$. 
Proof. By using Corollary 3.6 twice, one obtains

$g\left(R_{\phi_{\alpha} X \phi_{\alpha} Y} \phi_{\alpha} Z, \phi_{\alpha} W\right)=g\left(R_{X Y} Z, W\right)-P_{\alpha}\left(Z, W, X, \phi_{\alpha} Y\right)-P_{\alpha}\left(\phi_{\alpha} X, \phi_{\alpha} Y, Z, \phi_{\alpha} W\right)$.

Next, by using (3.2) and the property that $\phi_{\alpha}$ and $\psi_{\alpha}$ commute, we get that

$$
\begin{aligned}
P_{\alpha}\left(Z, W, X, \phi_{\alpha} Y\right)+P_{\alpha}\left(\phi_{\alpha} X, \phi_{\alpha} Y, Z, \phi_{\alpha} W\right)= & -\frac{c^{2}}{4}\left(\Psi_{\alpha}(Z, X) \Psi_{\alpha}\left(W, \psi_{\alpha} \phi_{\alpha} Y\right)\right. \\
& +\Psi_{\alpha}\left(Z, \psi_{\alpha} X\right) \Psi_{\alpha}\left(W, \psi_{\alpha} Y\right) \\
& +\Psi_{\alpha}\left(\phi_{\alpha} X, Z\right) \Psi_{\alpha}\left(\phi_{\alpha} Y, \psi_{\alpha} \phi_{\alpha} W\right) \\
& \left.+\Psi_{\alpha}\left(\phi_{\alpha} X, \psi_{\alpha} Z\right) \Psi_{\alpha}\left(\phi_{\alpha} Y, \phi_{\alpha} W\right)\right) .
\end{aligned}
$$

Thus the assertion follows.

We recall that on an almost contact metric manifold $(M, \phi, \xi, \eta, g)$ one defines a $\phi$-section as the 2-plane spanned by $X$ and $\phi X$, where $X$ is a unit vector field orthogonal to $\xi$. Then the sectional curvature $H(X):=K(X, \phi X)=g\left(R_{X \phi X} \phi X, X\right)$ is called $\phi$-sectional curvature. In a 3 -quasi-Sasakian manifold $M$, we denote by $H_{\alpha}$ the $\phi_{\alpha}$-sectional curvature.

Theorem 3.8. For any $X \in \Gamma(\mathcal{H})$ the $\phi_{\alpha}$-sectional curvatures of a 3-quasi-Sasakian manifold of rank $4 l+3$ satisfy the following relation

$$
H_{1}(X)+H_{2}(X)+H_{3}(X)=\frac{3 c^{2}}{4} g\left(X_{\mathcal{E}^{4 l}}, X_{\mathcal{E}^{4 l}}\right)^{2},
$$

where $X_{\mathcal{E}^{4 l}}$ denotes the projection of $X$ onto the distribution $\mathcal{E}^{4 l}$. In particular,

$$
H_{1}(X)+H_{2}(X)+H_{3}(X)= \begin{cases}\frac{3 c^{2}}{4}, & \text { for any } X \in \Gamma\left(\mathcal{E}^{4 l}\right) ; \\ 0, & \text { for any } X \in \Gamma\left(\mathcal{E}^{4 m}\right) .\end{cases}
$$

Proof. From Corollary 3.6 it follows that, for any $X, Y, Z, W \in \Gamma(\mathcal{H})$,

$$
\begin{aligned}
g\left(R_{X Y} \phi_{\alpha} Z, \phi_{\alpha} W\right)= & g\left(R_{X Y} Z, W\right)+\frac{c^{2}}{4}\left(\Psi_{\alpha}\left(Y, \psi_{\alpha} Z\right) g\left(\psi_{\alpha} X, \phi_{\alpha} W\right)\right. \\
& -\Psi_{\alpha}\left(X, \psi_{\alpha} Z\right) g\left(\psi_{\alpha} Y, \phi_{\alpha} W\right)-\Psi_{\alpha}(Y, Z) g\left(\psi_{\alpha}^{2} X, \phi_{\alpha} W\right) \\
& \left.+\Psi_{\alpha}(X, Z) g\left(\psi_{\alpha}^{2} Y, \phi_{\alpha} W\right)\right) .
\end{aligned}
$$

In (3.9) we put $\alpha=1, Z=X$ and $Y=W=\phi_{3} X$, getting

$$
\begin{aligned}
-g\left(R_{X \phi_{3} X} \phi_{1} X, \phi_{2} X\right)= & g\left(R_{X \phi_{3} X} X, \phi_{3} X\right)+\frac{c^{2}}{4}\left(-g\left(\phi_{3} X, \psi_{1}^{2} X\right) g\left(\psi_{1} X, \phi_{2} X\right)\right. \\
& +g\left(X, \psi_{1}^{2} X\right) g\left(\psi_{1} \phi_{3} X, \phi_{2} X\right)+g\left(\phi_{3} X, \psi_{1} X\right) g\left(\psi_{1}^{2} X, \phi_{2} X\right) \\
& \left.-g\left(X, \psi_{1} X\right) g\left(\psi_{1}^{2} \phi_{3} X, \phi_{2} X\right)\right) .
\end{aligned}
$$

By using the definition of the operators $\psi_{\alpha}$ and the property that $g\left(\phi_{\alpha} \cdot, \cdot\right)=$ $-g\left(\cdot, \phi_{\alpha} \cdot\right)$, one proves that $g\left(\psi_{1} X, \phi_{2} X\right), g\left(\phi_{3} X, \psi_{1} X\right)$, and $g\left(X, \psi_{1} X\right)$ vanish. Hence the previous relation becomes

$$
-g\left(R_{X \phi_{3} X} \phi_{1} X, \phi_{2} X\right)=g\left(R_{X \phi_{3} X} X, \phi_{3} X\right)+\frac{c^{2}}{4} g\left(X, \psi_{1}^{2} X\right) g\left(\psi_{1} \phi_{3} X, \phi_{2} X\right)
$$

since $g\left(X, \psi_{1}^{2} X\right) g\left(\psi_{1} \phi_{3} X, \phi_{2} X\right)=-g\left(X, \phi_{1}^{2} X_{\mathcal{E}^{4 l}}\right) g\left(\phi_{2} X_{\mathcal{E}^{4 l}}, \phi_{2} X\right)=g\left(X_{\mathcal{E}^{4 l}}, X\right)^{2}=$ $g\left(X_{\mathcal{E}^{4 l}}, X_{\mathcal{E}^{4 l}}\right)^{2}$. Making cyclic permutations of $\{1,2,3\}$, one gets

$$
\begin{aligned}
& -g\left(R_{X \phi_{1} X} \phi_{2} X, \phi_{3} X\right)=-H_{1}(X)+\frac{c^{2}}{4} g\left(X_{\mathcal{E}^{4 l}}, X_{\mathcal{E}^{4 l}}\right)^{2} \\
& -g\left(R_{X \phi_{2} X} \phi_{3} X, \phi_{1} X\right)=-H_{2}(X)+\frac{c^{2}}{4} g\left(X_{\mathcal{E}^{4 l}}, X_{\mathcal{E}^{4 l}}\right)^{2} .
\end{aligned}
$$


Then by summing (3.10), (3.11), (3.12), the claim follows from the Bianchi identity.

The notion of horizontal sectional curvature ([7]) plays in the context of 3structures the same role played by the $\phi$-sectional curvature in contact metric geometry. Let $\left(\phi_{\alpha}, \xi_{\alpha}, \eta_{\alpha}, g\right)$ be an almost contact 3 -structure on $M$. Let $X$ be a horizontal vector at a point $x$. Then one can consider the 4-dimensional subspace $\mathcal{H}_{x}(X)$ of $T_{x} M$ defined by $\mathcal{H}_{x}(X)=\left\langle X, \phi_{1} X, \phi_{2} X, \phi_{3} X\right\rangle . \mathcal{H}_{x}(X)$ is called the horizontal section determined by $X$. If the sectional curvature for any two vectors belonging to $\mathcal{H}_{x}(X)$ is a constant $k(X)$ depending only upon the fixed horizontal vector $X$ at $x$, then $k(X)$ is said to be the horizontal sectional curvature with respect to $X$ at $x$. Now let $X$ be an arbitrary horizontal vector field on $M$. If the horizontal section $\mathcal{H}_{x}(X)$ at any point $x$ of $M$ has a horizontal sectional curvature whose value $k(X)$ is independent of $X$, we say that the manifold $M$ is of constant horizontal sectional curvature at $x$. It is known ([7]) that a 3 -Sasakian manifold has constant horizontal sectional curvature if and only if it has constant curvature 1. We now consider the 3-quasi-Sasakian setting.

Theorem 3.9. A 3-quasi-Sasakian manifold has constant horizontal sectional curvature if and only if it is either 3-c-Sasakian or 3-cosymplectic. In the first case it is a space of constant curvature $c^{2} / 4$, in the latter it is flat.

Proof. We distinguish the case when $M$ is 3-cosymplectic and $M$ is 3-quasi-Sasakian of rank $4 l+3$. Let $M$ be a 3 -cosymplectic manifold of constant horizontal sectional curvature $k$ and let $x$ be a point of $M$. There exists a local Riemannian submersion $\pi$ defined on an open neighborhood of $x$ with base space a hyper-Kähler manifold $\left(M^{\prime}, J_{\alpha}^{\prime}, g^{\prime}\right)$. We recall the well-known O'Neill formula ([11]) relating the sectional curvatures of the total and base spaces

$$
K(Y, Z)=K^{\prime}(Y, Z)-3\left\|A_{Y} Z\right\|=K^{\prime}(Y, Z),
$$

$A$ denoting the O'Neill tensor, which in this case vanishes identically since the distribution $\mathcal{H}$ is integrable. As the value of $k$ does not depend of the horizontal section $\mathcal{H}_{x}(X)$ at $x$, we can choose $X$ to be a basic vector field. Since for any $\alpha, \beta \in\{1,2,3\}, \mathcal{L}_{\xi_{\alpha}} \phi_{\beta}=0, \mathcal{H}_{x}(X)$ projects to a horizontal section $\mathcal{H}_{x^{\prime}}\left(X^{\prime}\right)$ on $x^{\prime}=\pi(x)$. Then, (3.13) implies that $M^{\prime}$ has constant horizontal sectional curvature $k$. It is well known that a hyper-Kähler manifold of constant horizontal sectional curvature is flat, hence by using (3.13) again we get that $M$ is horizontally flat. On the other hand, for any $Z \in \Gamma(T M)$, we have $K\left(Z, \xi_{\alpha}\right)=0$ (cf. [5, Lemma 2]). Thus $M$ is flat. Let us now suppose that $M$ is a 3 -quasi-Sasakian manifold of rank $4 l+3$ with constant horizontal sectional curvature $k$. By definition of horizontal sectional curvature, $k=k(X)=H_{1}(X)=H_{2}(X)=H_{3}(X)$. Suppose the rank of $M$ is not maximal, that is $\mathcal{E}^{4 l}$ does not coincide with $\mathcal{H}$. Then, from (3.8), we get that $k(X)=\frac{c^{2}}{4}$ for $X \in \Gamma\left(\mathcal{E}^{4 l}\right)$ and $k(X)=0$ for $X \in \Gamma\left(\mathcal{E}^{4 m}\right)$. This is in contrast with the fact that the value of $k$ does not depend of $X$. Thus $M$ is necessarily of maximal rank and $k=\frac{c^{2}}{4}$. Hence, due to [4, Corollary 4.4], $M$ is 3-c-Sasakian. Observe now that one can apply a homothety to the given structure, that is a change of the structure tensors of the following type

$$
\bar{\phi}_{\alpha}:=\phi_{\alpha}, \quad \bar{\xi}_{\alpha}:=\frac{2}{c} \xi_{\alpha}, \quad \bar{\eta}_{\alpha}:=\frac{c}{2} \eta_{\alpha}, \quad \bar{g}:=\frac{c^{2}}{4} g,
$$

Then it is easy to check that the resulting structure $\left(\bar{\phi}_{\alpha}, \bar{\xi}_{\alpha}, \bar{\eta}_{\alpha}, \bar{g}\right)$ is 3-Sasakian and its horizontal sectional curvature is proportional to that of $\left(\phi_{\alpha}, \xi_{\alpha}, \eta_{\alpha}, g\right)$. Therefore, due to [7], $\left(M, \bar{\phi}_{\alpha}, \bar{\xi}_{\alpha}, \bar{\eta}_{\alpha}, \bar{g}\right)$ is a space of constant sectional curvature and therefore the same is true for $\left(M, \phi_{\alpha}, \xi_{\alpha}, \eta_{\alpha}, g\right)$. Its sectional curvature is $k=\frac{c^{2}}{4}$. 


\section{REFERENCES}

[1] D. E. Blair, Riemannian geometry of contact and symplectic manifolds, Progress in Mathematics, vol. 203, Birkhäuser Boston Inc., Boston, MA, 2010.

[2] D. E. Blair, The theory of quasi-Sasakian structures, J. Diff. Geom. 1 (1967), 331-345.

[3] B. Cappelletti Montano, A. De Nicola, G. Dileo, 3-quasi-Sasakian manifolds, Ann. Glob. Anal. Geom. 33 (2008) 397-409.

[4] B. Cappelletti Montano, A. De Nicola, G. Dileo, The geometry of 3-quasi-Sasakian manifolds, Internat. J. Math. 20 (2009), 1081-1105.

[5] S. Goldberg, K. Yano, Integrability of almost cosymplectic manifolds, Pacific J. Math. 31 (1969), 373-382.

[6] T. Kashiwada, F. Martin Cabrera, M. M. Tripathi, Non-existence of certain 3-structures, Rocky Mountain J. Math. 35 (2005), 1953-1979.

[7] M. Konishi, S. Funabashi, On Riemannian manifolds with Sasakian 3-structure of constant horizontal sectional curvature, Kodai Math. Sem. Rep. 27 (1976), 362-366.

[8] Y. Y. Kuo, On almost 3-structure, Tôhoku Math. J. 22 (1970), 325-332.

[9] D. Janssens, L. Vanhecke, Almost contact structures and curvature tensors, Kodai Math. J. 4 (1981), 127.

[10] Z. Olszak, Curvature properties of quasi-Sasakian manifolds, Tensor 38 (1982), 19-28.

[11] B. O'Neill, The fundamental equations of a submersion, Mich. Math. J. 13 (1966), 459-469.

[12] A. R. Rustanov, On the geometry of quasi-Sasakian manifolds, Uspekhi Mat. Nauk. 49 (1994), 221-222; translation in Russian Math. Surveys 49 (1994), 243-244.

[13] S. Tanno, Quasi-Sasakian structures of rank $2 p+1$, J. Diff. Geom. 5 (1971), 317-324.

[14] C. Udriste, Structures presque coquaternioniennes, Bull. Math. Soc. Sci. Math. R. S. Roumanie 13 (1969), 487-507.

Dipartimento di Matematica e Informatica, Università degli Studi di Cagliari, Via Ospedale 72, 09124 Cagliari, Italy

E-mail address: b.cappellettimontano@gmail.com

CMuC, Department of Mathematics, University of Coimbra, 3001-454 Coimbra, PorTUGAL

E-mail address: antondenicola@gmail.com

CMUC, Department of Mathematics, University of Coimbra, 3001-454 Coimbra, PorTUGAL

E-mail address: yudin@mat.uc.pt 